\title{
Determination of the Weibull parameters from the mean value and the coefficient of variation of the measured strength for brittle ceramics
}

\author{
Bin DENG ${ }^{a}$, Danyu JIANG ${ }^{b, *}$ \\ ${ }^{a}$ Department of the Prosthodontics, Chinese PLA General Hospital, Beijing 100853, China \\ ${ }^{b}$ Analysis and Testing Center for Inorganic Materials, State Key Laboratory of High Performance Ceramics and \\ Superfine Microstructure, Shanghai Institute of Ceramics, Shanghai 200050, China
}

Received: January 11, 2017; Accepted: April 7, 2017

(C) The Author(s) 2017. This article is published with open access at Springerlink.com

\begin{abstract}
Accurate estimation of Weibull parameters is an important issue for the characterization of the strength variability of brittle ceramics with Weibull statistics. In this paper, a simple method was established for the determination of the Weibull parameters, Weibull modulus $m$ and scale parameter $\sigma_{0}$, based on Monte Carlo simulation. It was shown that an unbiased estimation for Weibull modulus can be yielded directly from the coefficient of variation of the considered strength sample. Furthermore, using the yielded Weibull estimator and the mean value of the strength in the considered sample, the scale parameter $\sigma_{0}$ can also be estimated accurately.
\end{abstract}

Keywords: Weibull distribution; Weibull parameters; strength variability; unbiased estimation; coefficient of variation

\section{Introduction}

Weibull statistics [1] has been widely employed to model the variability in the fracture strength of brittle ceramics [2-8]. In general, a two-parameter form of the Weibull function is adopted to give the cumulative failure probability, $P$, of a component or specimen at a given applied stress, $\sigma$, as [2]:

$$
P=1-\exp \left[-\left(\frac{\sigma}{\sigma_{0}}\right)^{m}\right]
$$

where $m$ is called the Weibull modulus and $\sigma_{0}$ is the scale parameter with the same dimension as $\sigma$.

Different methods have been used to estimate the Weibull parameters, $m$ and $\sigma_{0}$, the most popular being the least-square (LS) method and the maximum-

* Corresponding author.

E-mail: dyjiang@mail.sic.ac.cn likelihood (ML) method. Many studies [9-18] based on Monte Carlo simulation have shown that each of these methods has its benefits and drawbacks.

According to the theory of statistics, the expected value, $E(\sigma)$, and the variance, $D(\sigma)$, of the twoparameter Weibull distribution can be expressed as [19]:

$$
\begin{gathered}
E(\sigma)=\sigma_{0} \Gamma\left(1+\frac{1}{m}\right) \\
D(\sigma)=\sigma_{0}^{2}\left[\Gamma\left(1+\frac{2}{m}\right)-\Gamma^{2}\left(1+\frac{1}{m}\right)\right]
\end{gathered}
$$

Thus, the coefficient of variation for this distribution, $\left(c_{\mathrm{v}}\right)_{\mathrm{w}}$, is

$$
\left(c_{\mathrm{v}}\right)_{\mathrm{w}}=\frac{\sqrt{D(\sigma)}}{E(\sigma)}=\frac{\left[\Gamma\left(1+\frac{2}{m}\right)-\Gamma^{2}\left(1+\frac{1}{m}\right)\right]^{1 / 2}}{\Gamma\left(1+\frac{1}{m}\right)}
$$

Equation (4) shows that $\left(c_{\mathrm{v}}\right)_{\mathrm{w}}$ is dependent only on the Weibull modulus, $m$, and decreases as $m$ increases 
when $m>1$. Based on this relation, Gong et al. [20,21] proposed an empirical equation for determining the Weibull modulus, $m_{\mathrm{es}}$, from the coefficient of variation, $\left(c_{\mathrm{v}}\right)_{\mathrm{f}}$, of measured fracture strength:

$$
m_{\mathrm{es}}=\frac{\alpha}{\left(c_{\mathrm{v}}\right)_{\mathrm{f}}}+\beta
$$

where $\alpha$ and $\beta$ are both constants dependent on the sample size, and were determined and formulated by analyzing the data produced by Monte Carlo simulation in the original works of Gong et al. [20,21].

It should be noted that Gong et al.'s method is only for the determination of Weibull modulus, and the other Weibull parameter, the scale parameter $\sigma_{0}$, should be determined still with the conventional methods, i.e., the LS method or the ML method. Undoubtedly, this drawback would impede the application of this simple method.

The aim of this paper is to contribute supplementary information towards a more accurate determination of the Weibull modulus based on Eq. (5) and then establish an approach for the direct determination of the scale parameter $\sigma_{0}$. Considering its versatility and simplicity, the LS method was employed in the present work to yield the referenced Weibull parameters for the analyses and comparison.

\section{Monte Carlo simulation procedure}

The basic idea of the Monte Carlo simulation can be described as: suppose we have a material whose fracture strength variation follows a two-parameter distribution of known parameters, $m_{\mathrm{tr}}$ and $\left(\sigma_{0}\right)_{\mathrm{tr}}$ (for the sake of simplicity, $\sigma_{0}$ is set to be unity throughout the whole study); thus we can choose $N$ random specimens of this material to yield a sample, "measure" the fracture strength of each specimen by assigning a random number in the internal of $0-1$ as its corresponding fracture probability, $P$, and then estimate the Weibull parameters.

In continuation of this idea, a computer program is written to establish the relationships between the estimated Weibull parameters, $m_{\mathrm{es}}$ and $\left(\sigma_{0}\right)_{\mathrm{es}}$, the mean values, $\bar{\sigma}_{\mathrm{f}}$, and the coefficient of variation, $\left(c_{\mathrm{v}}\right)_{\mathrm{f}}$, of the "measured" fracture strengths.

(i) Firstly, a sample of random number, $\operatorname{RND}_{i}(i=1$, $2, \ldots, N)$ in the interval of $0-1$ is produced to calculate the strength value, $\sigma_{i}$, with the prescribed $m_{\mathrm{tr}}$ :

$$
\sigma_{i}=\left[\ln \left(\frac{1}{1-\mathrm{RND}_{i}}\right)\right]^{1 / m_{\mathrm{tr}}}
$$

Thus, a sample containing $N$ strength values, $\sigma_{1}$, $\sigma_{2}, \ldots, \sigma_{N}$, is obtained.

(ii) The yielded strength values are ordered from the lowest to the highest and the $i$ th result in the set of $N$ strength data, $\sigma_{i}$, is assigned a cumulative fracture probability, $P_{i}$, which is calculated with [22-24]:

$$
P_{i}=\frac{i-0.5}{N}
$$

The strengths and the probabilities are then analyzed, by using a simple, least-square regression method, according to the alternative form of Eq. (1):

$$
\ln \ln \left(\frac{1}{1-P_{i}}\right)=m_{\mathrm{es}} \ln \sigma-m_{\mathrm{es}} \ln \left(\sigma_{0}\right)_{\mathrm{es}}
$$

to give the estimated values of the Weibull parameters, $m_{\mathrm{es}}$ and $\left(\sigma_{0}\right)_{\mathrm{es}}$.

(iii) The mean values, $\bar{\sigma}_{\mathrm{f}}$, and the standard deviation, $S_{\mathrm{f}}$, of the $N$ strength data are calculated as

$$
\begin{gathered}
\bar{\sigma}_{\mathrm{f}}=\frac{1}{N} \sum_{i=1}^{N} \sigma_{i} \\
S_{\mathrm{f}}^{2}=\frac{1}{N} \sum_{i=1}^{N}\left(\sigma_{i}-\bar{\sigma}_{\mathrm{f}}\right)^{2}
\end{gathered}
$$

Then coefficient of variation, $\left(c_{\mathrm{v}}\right)_{\mathrm{f}}$, is given by

$$
\left(c_{\mathrm{v}}\right)_{\mathrm{f}}=\frac{S_{\mathrm{f}}}{\bar{\sigma}_{\mathrm{f}}}
$$

To study the effect of sample size, $N$ is increased progressively from 10 to 200 . Similarly, $m_{\text {tr }}$ is increased progressively from 5 to 25 to study the effect of Weibull modulus. For each set of given sample size, $N$, and the prescribed Weibull modulus, $m_{\text {tr }}$, the above-mentioned procedure is repeated for 100000 times in order to ensure statistical convergence of the results.

\section{Results and discussion}

\section{1 Refinement of Gong et al.'s method}

In the original works of Gong et al. [20,21], by repeating the above-mentioned Monte Carlo simulation procedure 4000 times for a given $N$ and $m_{\text {tr }}=2,3,4, \ldots$, $25,26,100000(=4000 \times 25)$ samples of strength data 
were produced. Then, a least-square regression analysis was applied with all the resultant 100000 estimated parameters, $m_{\mathrm{es}}$ and $\left(c_{\mathrm{v}}\right)_{\mathrm{f}}$, to yield the parameters $\alpha$ and $\beta$ in Eq. (5). Such a treatment, however, seems to be questioned.

Figure 1 shows, as an example, some data produced with $N=30$ and $m_{\mathrm{tr}}=2.5,10,20$. It can be seen from the insert, which shows the enlargement of the small $m_{\mathrm{tr}}$ region, that the data points produced with $m_{\mathrm{tr}}=2.5$ show large scatter, making it unreliable to fit these data to a straight line. The dashed line in Fig. 1 is obtained by least-square regression analysis of the data produced with $m_{\mathrm{tr}}=20$. Clearly, most of the data points corresponding to $m_{\mathrm{tr}}=2.5$ locate above the dashed line. If all the data shown in Fig. 1 were fitted according to Eq. (5), however, most of the data points corresponding to $m_{\mathrm{tr}}=2.5$ locate below the best-fit line (solid line in Fig. 1).

To get a clearer understanding on the experimental phenomena shown in Fig. 1, 100000 samples of strength data were produced with given $N$ and $m_{\text {tr }}$. Then the resultant data were analyzed according to Eq. (5) to yield $\alpha$ and $\beta$. Figure 2 shows the best-fit value of the parameter $\alpha$ as a function of the prescribed $m_{\text {tr }}$ for some selected $N$. As can be seen, $\alpha$ strongly depends on the prescribed $m_{\mathrm{tr}}$ value, especially when the sample size $N$ is smaller. On the other hand, the best-fit value of the parameter $\beta$ is found to be very small, less than $5 \times 10^{-5}$ in each case. These results imply that, each set of the $m_{\mathrm{es}}-\left(c_{\mathrm{v}}\right)_{\mathrm{f}}$ data points produced with a given $m_{\mathrm{tr}}$ would fall along a straight line starting from the origin and with a slope dependent of $m_{\mathrm{tr}}$. From the viewpoint

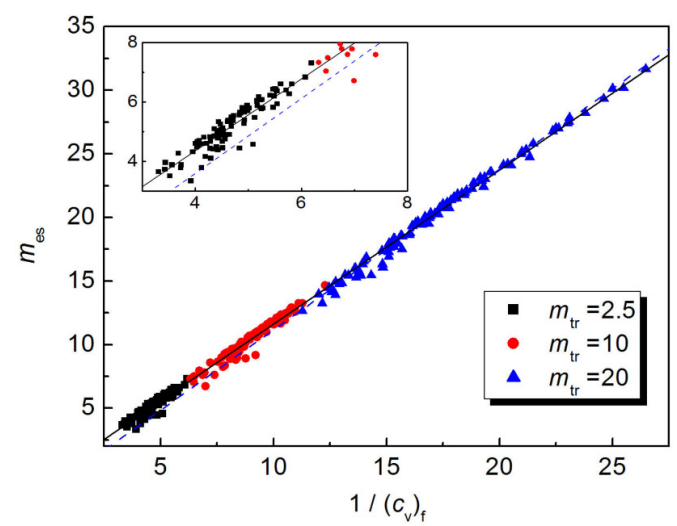

Fig. 1 Variation of $m_{\mathrm{es}}$ with $\left(c_{\mathrm{v}}\right)_{\mathrm{f}}$. Data produced with $N=30$ and $m_{\mathrm{tr}}=2.5,10,25$. Solid line: best-fit result of all the data; dashed line: best-fit result of only the data produced with $m_{\mathrm{tr}}=20$.

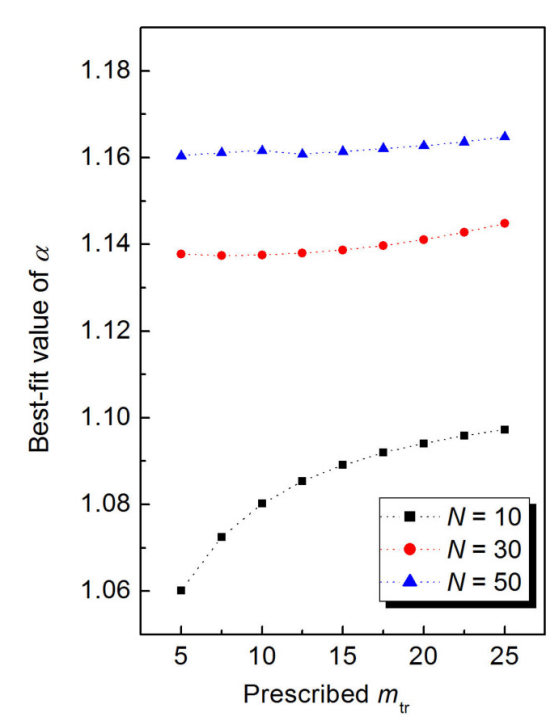

Fig. 2 Best-fit value of $\alpha$ as a function of the prescribed $m_{\mathrm{tr}}$.

of statistics, fitting the data produced with different $m_{\text {tr }}$ to a single straight line would result in some uncertainties in the best-fit values of $\alpha$ and $\beta$.

As mentioned above, in the original works of Gong et al. [20,21], the regression analyses for determining $\alpha$ and $\beta$ were conducted within $m_{\text {tr }}$ ranging from 2 to 26 and only 4000 data produced with each prescribed $m_{\text {tr }}$ were used. Based on the above discussion, such a simulation procedure seems to be insufficient to ensure the statistical convergence and warrant a further improvement.

Therefore, three key issues were considered here to improve Gong et al.'s simulation procedure:

(i) For each given set of $N$ and $m_{\text {tr }}, 100000$ samples were analyzed to ensure the statistical convergence.

(ii) Noting that the Weibull modulus has typical values ranging 5 to 20 for brittle ceramics, the smallest and the largest $m_{\text {tr }}$ were set to be 5 and 25 , respectively.

(iii) For a given $N$, the mean values of $m_{\mathrm{es}}$ and $\left(c_{\mathrm{v}}\right)_{\mathrm{f}}$ corresponding to each prescribed $m_{\mathrm{tr}}$, were employed for the regression analyses for determining the parameters $\alpha$ and $\beta$.

The relationships between the mean values of $m_{\mathrm{es}}$ and $\left(c_{\mathrm{v}}\right)_{\mathrm{f}}$ for different sample size, $N$, are now shown in Fig. 3. Also shown in Fig. 3 are the best-fit lines obtained by analyzing the data according to Eq. (5). It can be seen that, for each given sample size, $N$, an excellent linear relationship between $m_{\mathrm{es}}$ and $\left(c_{\mathrm{v}}\right)_{\mathrm{f}}$ is obtained and, in each case, the regression analysis 


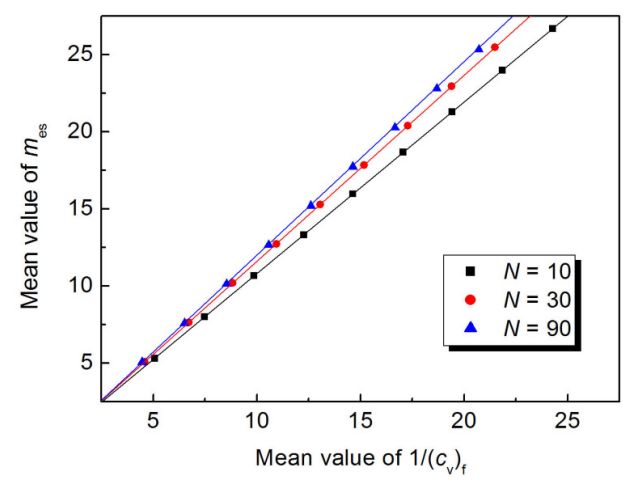

Fig. 3 Variations of the mean value of $m_{\mathrm{es}}$ with the mean value of $\left(c_{\mathrm{v}}\right)_{\mathrm{f}}$ for different sample size.

returns a correlation coefficient of unity.

The best-fit values of the parameters $\alpha$ and $\beta$ are shown in Fig. 4. Similar to the results obtained in the original work of Gong and $\mathrm{Li}$ [21], the sample-size dependence of $\alpha$ and $\beta$ can be respectively described with the following empirical equations:

$$
\begin{gathered}
\alpha=1.26-0.26 \exp \left(-\frac{N}{8.21}\right)-0.09 \exp \left(-\frac{N}{49.78}\right)(12) \\
\beta=-0.54+0.38 \exp \left(-\frac{N}{8.98}\right)+0.14 \exp \left(-\frac{N}{48.25}\right)
\end{gathered}
$$

The results given in Eqs. (12) and (13) are only slightly different from those reported by Gong and $\mathrm{Li}$ [21]. But it is clear, based on the above discussion, that Eqs. (12) and (13) would yield more accurate results for the determination of the parameters $\alpha$ and $\beta$.

\section{2 Statistical comparison of the estimated Weibull parameters}

It is necessary to make a statistical comparison between the two estimators, one calculated directly from Eq. (5) (denoted as $m_{1}$ ) and the other estimated by the conventional LS method (denoted as $m_{2}$ ).

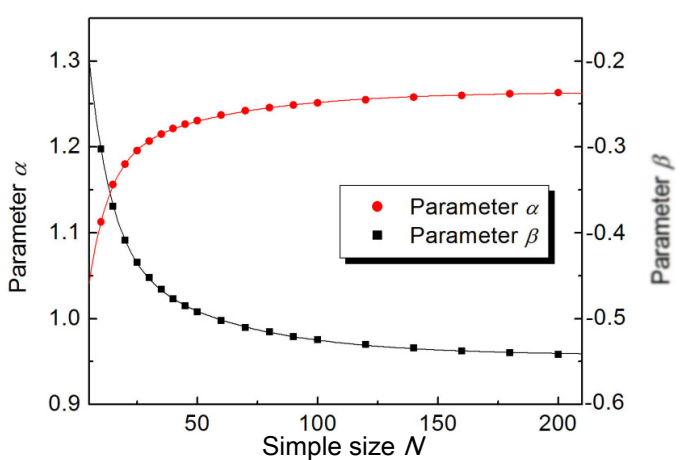

Fig. 4 Variations of the best-fit values of the parameters $\alpha$ and $\beta$ with sample size.
Figure 5 shows the mean value and the standard deviation of the $100000 \mathrm{~m}_{2} / \mathrm{m}_{\mathrm{tr}}$ as functions of the prescribed $m_{\text {tr }}$. As can be seen, within the examined range of $5 \leqslant m_{\text {tr }} \leqslant 25$, both the mean value and the standard deviation of $m_{\mathrm{es}} / m_{\mathrm{tr}}$ are independent of $m_{\mathrm{tr}}$, showing only a sample-size dependence.

In Fig. 6, the mean value and the standard deviation of the $100000 m_{1} / m_{\text {tr }}$ and $m_{2} / m_{\text {tr }}$ estimated with $m_{\mathrm{tr}}=10$ are shown as functions of the sample size, $N$. Both mean values are identical with each other, implying that the simple method proposed in the present study would give a Weibull estimator comparable to

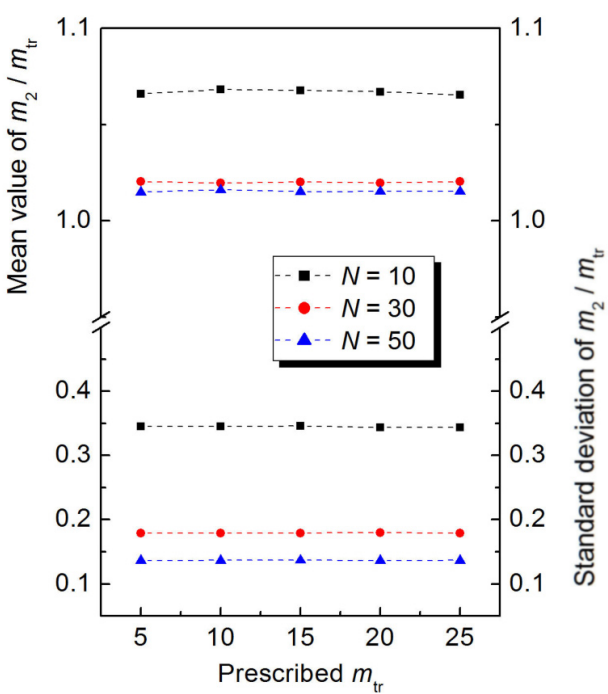

Fig. 5 Variations of the mean value and the standard deviation of $m_{\mathrm{es}} / m_{\mathrm{tr}}$ estimated with the conventional LS method with the prescribed $m_{\mathrm{tr}}$.

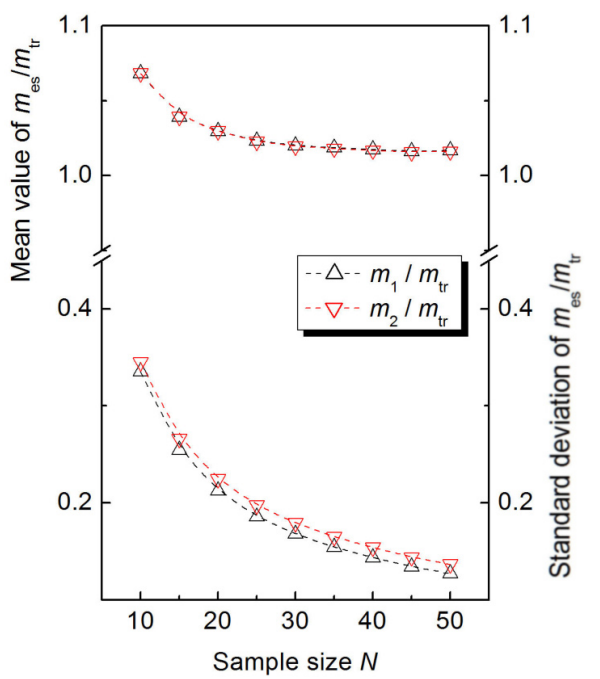

Fig. 6 Sample-size dependence of the mean value and the standard deviation of $m_{\mathrm{es}} / m_{\mathrm{tr}}$ estimated with the simple method and the LS method $\left(m_{\mathrm{tr}}=10\right)$. 
that estimated with the conventional LS method. Note that the standard deviation of $m_{1}$ is slightly smaller than that of $m_{2}$. One can expect that the statistical distribution of $m_{1}$ would be somewhat narrower than that of $m_{2}$.

Figure 7 shows as examples the empirical probability density functions of $m_{\mathrm{es}} / m_{\mathrm{tr}}$ estimated with the simple method and the LS method. In constructing these plots, the resultant $100000 m_{\mathrm{es}} / m_{\mathrm{tr}}$ with $m_{\mathrm{tr}}=10$ were classified into groups that fell into definite intervals. The number of estimators that fell into each interval was countered and normalized through division by 100000 to produce the relative frequency of occurrence, the $y$-value, and the $x$-value is simply the midpoint of the given interval. Clearly, there is no distinct statistical difference between $m_{1} / m_{\mathrm{tr}}$ and $m_{2} / m_{\text {tr }}$, indicating that the simple method proposed in the present study is suitable for the determination of Weibull modulus.

\section{3 Determination of the scale parameter}

In the original works of Gong et al. [20,21], no
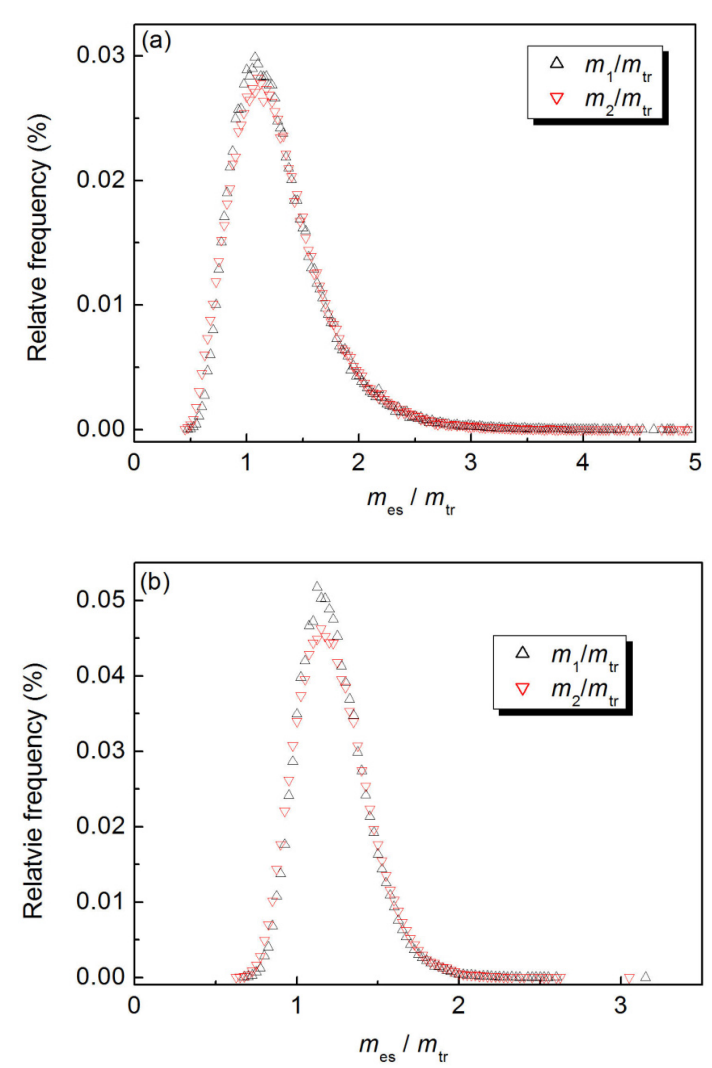

Fig. 7 Empirical probability density functions of $m_{\text {es }} / m_{\text {tr }}$ estimated with the simple method and the LS method: (a) $N=10$; (b) $N=30$. discussion was made for the determination of the scale parameter, $\sigma_{0}$. Even the Weibull modulus can be determined directly with the coefficient of variation of the strength sample, another method, e.g., LS method or ML method, is still required to estimate the scale parameter. This is one of the main drawbacks which may impede the application of this simple method.

Now we try to establish an approach for the direct determination of the scale parameter $\sigma_{0}$. Similar to the determination of Weibull modulus, we start with the expression of the expected value of the two-parameter Weibull modulus, Eq. (2).

As shown in Fig. 8, for $x$ ranging from 1.0 to 1.4, $\Gamma(x)$ can be approximately expressed as a polynomial form:

$\Gamma(x)=3.5977-6.0676 x+5.2886 x^{2}-2.1918 x^{3}+0.3730 x^{4}$

Thus, based on Eq. (2), the estimated value of $\sigma_{0}$, $\left(\sigma_{0}\right)_{\text {es }}$, for a given sample of strength data can be approximately determined with

$$
\left(\sigma_{0}\right)_{\mathrm{es}}=\bar{\sigma}_{\mathrm{f}} / \Gamma\left(1+\frac{1}{m_{\mathrm{es}}}\right)
$$

where $\bar{\sigma}_{\mathrm{f}}$ is the mean value of the measured strength and $m_{\mathrm{es}}$ is calculated with Eq. (11). Once $m_{\mathrm{es}}$ is obtained from Eq. (5), the $\Gamma$ function in Eq. (15) can be calculated with Eq. (14).

Similarly, hereafter the $\left(\sigma_{0}\right)_{\mathrm{es}}$ determined with Eqs. (14) and (15) is denoted as $\left(\sigma_{0}\right)_{1}$ and the $\left(\sigma_{0}\right)_{\mathrm{es}}$ estimated by the conventional LS method is denoted as $\left(\sigma_{0}\right)_{2}$.

The efficiency of Eqs. (14) and (15) in determining $\left(\sigma_{0}\right)_{\mathrm{es}}$ can be verified by the Monte Carlo simulation. Figure 9 shows the mean value and the standard deviation of the $100000\left(\sigma_{0}\right)_{1}$ and $\left(\sigma_{0}\right)_{2}$ estimated

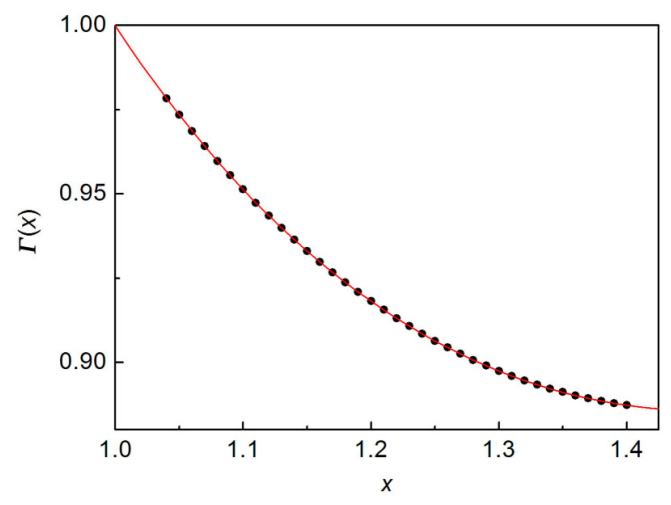

Fig. $8 \Gamma(x)$ as a function of $x$. Symbol: individual $\Gamma(x)$ value; line: the best-fit result of the data points. 


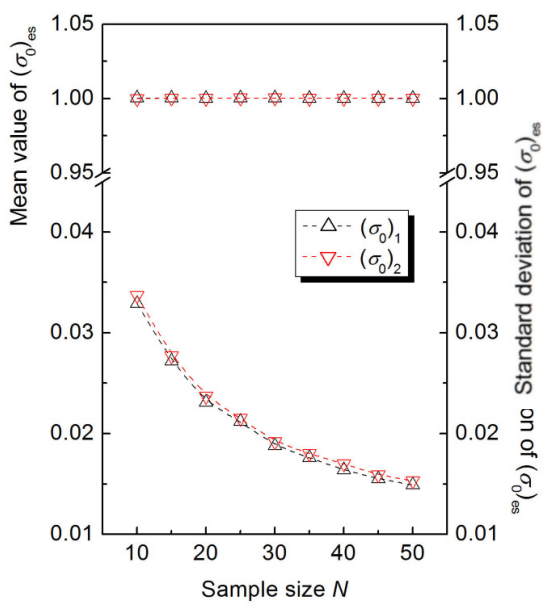

Fig. 9 Sample-size dependence of the mean value and the standard deviation of $\left(\sigma_{0}\right)_{\mathrm{es}}$ estimated with the simple method and the LS method $\left(m_{\mathrm{tr}}=10\right)$.

with $m_{\mathrm{tr}}=10$ as functions of the sample size, $N$. Similar to the Weibull modulus shown in Fig. 6, the mean values of $\left(\sigma_{0}\right)_{1}$ and $\left(\sigma_{0}\right)_{2}$ are identical with each other and the standard deviation of $\left(\sigma_{0}\right)_{1}$ is slightly smaller than that of $\left(\sigma_{0}\right)_{2}$.

Although Monte Carlo simulation has been widely used to evaluate the statistical properties of Weibull modulus estimated by different methods [9-18], only a little effort $[10,12]$ has been devoted to the statistical properties of the estimated scale parameter $\sigma_{0}$. This is because that, for each conventional point-estimation method, no significant variation occurs in determining $\sigma_{0}$ [12]. Figure 10 shows the empirical probability density functions for $\left(\sigma_{0}\right)_{2}$ estimated with different $m_{\text {tr }}$ and different sample size $N$. Clearly, the mean value of the estimated $\sigma_{0}$ is nearly independent of the prescribed $m_{\mathrm{tr}}$ and the sample size $N$. Increases in $m_{\mathrm{tr}}$ or $N$ would only narrow the dispersion of the estimated scale parameter. These results, combining with the results shown in Fig. 9, indicate that, for the conventional point-estimation methods such as LS method, $\sigma_{0}$ can be estimated with a higher degree of accuracy than $m$.

Figure 11 compares the empirical probability density curves of $\left(\sigma_{0}\right)_{1}$ and $\left(\sigma_{0}\right)_{2}$ for selected $m_{\mathrm{tr}}$ and $N$. There is no distinct statistical difference between $\left(\sigma_{0}\right)_{1}$ and $\left(\sigma_{0}\right)_{2}$, indicating that the simple method proposed in the present study is suitable for the determination of scale parameters.

\section{4 Examples of application}

It is interesting to examine the joint effect of the
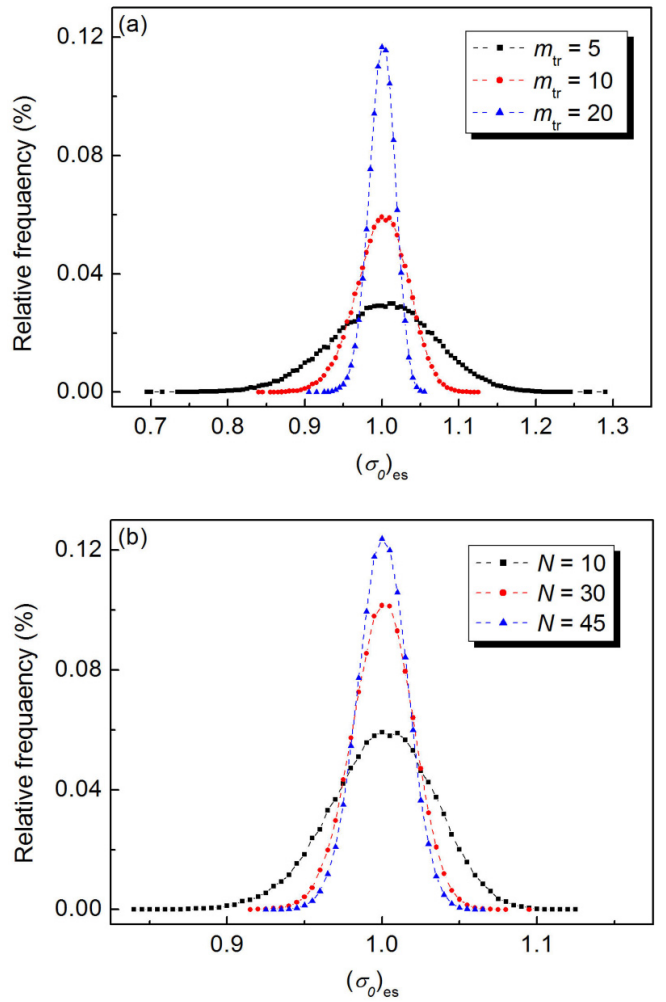

Fig. 10 Empirical probability density functions of $\left(\sigma_{0}\right)_{\mathrm{es}}$ estimated with the LS method: (a) $N=30$; (b) $m_{\mathrm{tr}}=10$.
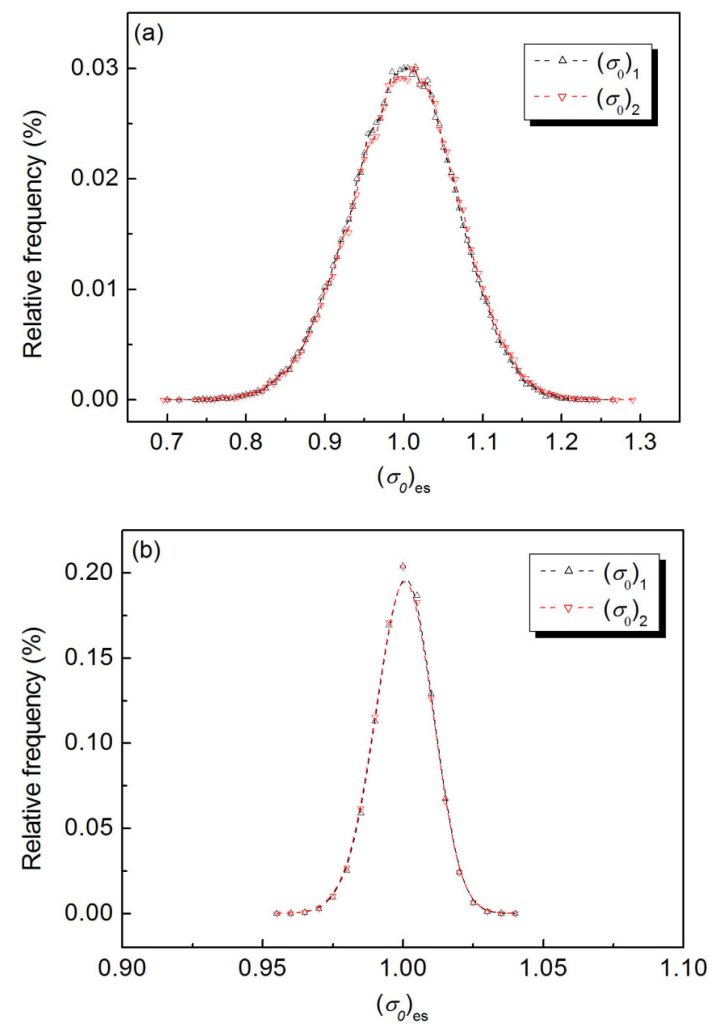

Fig. 11 Comparisons of the empirical probability density curves of $\left(\sigma_{0}\right)_{1}$ and $\left(\sigma_{0}\right)_{2}:$ (a) $N=10, m_{\mathrm{tr}}=5$; (b) $N=20$, $m_{\mathrm{tr}}=20$. 
dispersion of estimated parameters, $m_{\mathrm{es}}$ and $\left(\sigma_{0}\right)_{\mathrm{es}}$, in the characterization of some real strength samples. For this purpose, three strength datasets were selected from literature. Dataset 1 was selected from Table 2 of Ref. [25] in which the strength of a sintered silicon nitride was measured in three-point bending with specimens of $3 \mathrm{~mm}$ in thickness and $4 \mathrm{~mm}$ in width. Dataset 2 was selected from Fig. 1 of Ref. [2] which listed the three-point bending strength measured for a sintered alumina available commercially (Coors grade AD-999). Dataset 3 was selected from Table 1 of Ref. [26], in which a total of 40 strength data were given for a brittle glass of unknown composition. All the strength data in each dataset are now shown in the form of Weibull graphs in Fig. 12 by assigning each strength datum a probability of fracture, $P_{\mathrm{f}}$, defined with Eq. (7).

Each dataset was analyzed with the simple method proposed in the present study and the conventional LS method to yield the Weibull parameters, $m_{\mathrm{es}}$ and $\left(\sigma_{0}\right)_{\text {es }}$. The results are listed in Table 1. For each dataset, there exists a good agreement between the two sets of estimated parameters, giving a sound support for the above discussion. Using these estimated values, the Weibull lines for each dataset can be drawn directly with Eq. (1). As can be seen in Fig. 12, the Weibull lines drawn based on the simple method are overlapped with those based on the conventional LS method.

Table 1 Estimated Weibull parameters for the three examined datasets

\begin{tabular}{cccccc}
\hline \multirow{2}{*}{ Dataset } & \multicolumn{2}{c}{ The simple method } & & \multicolumn{2}{c}{ The LS method } \\
\cline { 2 - 3 } \cline { 5 - 6 } & $m_{1}$ & $\left(\sigma_{0}\right)_{1}$ & & $m_{2}$ & $\left(\sigma_{0}\right)_{2}$ \\
\hline 1 & 11.03 & 515.8 & & 11.10 & 514.8 \\
2 & 10.01 & 466.6 & 10.21 & 466.1 \\
3 & 10.71 & 64.7 & & 10.86 & 64.7 \\
\hline
\end{tabular}

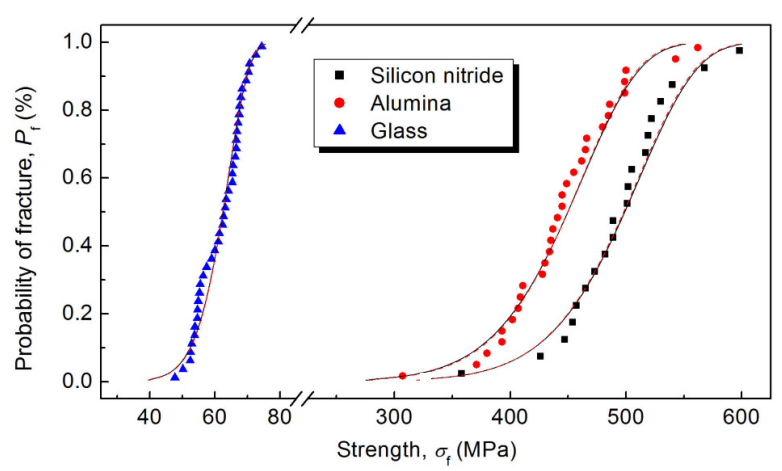

Fig. 12 Weibull graphs of the measured strength for $\mathrm{Si}_{3} \mathrm{~N}_{4}, \mathrm{Al}_{2} \mathrm{O}_{3}$, and glass. Solid lines and dashed lines are obtained by the simple method and the LS method, respectively.

\section{Conclusions}

Based on Monte Carlo simulation, a simple method was proposed in the present study for the determination of the Weibull parameters, $m$ and $\sigma_{0}$. This simple method can be summarized as

(i) An unbiased Weibull modulus, $m_{\mathrm{es}}$, can be calculated directly with Eq. (5) using the coefficient of variation, $\left(c_{\mathrm{v}}\right)_{\mathrm{f}}$, of the strength sample. The parameters included in Eq. (5) are shown in Fig. 4 and can also be calculated with Eqs. (12) and (13).

(ii) Using $m_{\mathrm{es}}$ calculated with Eq. (5) and the mean value of the strength in the considered sample, $\left(\sigma_{0}\right)_{\text {es }}$ can be obtained directly from Eq. (15). The resultant $\left(\sigma_{0}\right)_{\text {es }}$ is an unbiased estimation for the scale parameter $\sigma_{0}$.

Detailed analyses confirmed that both $m$ and $\sigma_{0}$ obtained by this simple method are statistically identical with those estimated with the conventional LS method. Especially, the standard deviations of both $m$ and $\sigma_{0}$ obtained by this simple method are slightly smaller than those estimated with the conventional LS method, implying that the simple method would yield a more accurate estimation of the Weibull parameters.

\section{Acknowledgements}

The authors acknowledge the support of Strategic Priority Research Program of the Chinese Academy of Sciences (Grant No. XDB06050301).

\section{References}

[1] Weibull W. A statistical distribution function of wide applicability. J Appl Mech 1951, 18: 293-297.

[2] Quinn G. Advanced structural ceramics: A round robin. J Am Ceram Soc 1990, 73: 2374-2384.

[3] Danzer R, Supancic P, Pascual J, et al. Fracture statistics of ceramics-Weibull statistics and deviations from Weibull statistics. Eng Fract Mech 2007, 74: 2919-2932.

[4] Quinn JB, Quinn GD. A practical and systematic review of Weibull statistics for reporting strengths of dental materials. Dent Mater 2010, 26: 135-147.

[5] Nohut S. Influence of sample size on strength distribution of advanced ceramics. Ceram Int 2014, 40: 4285-4295.

[6] Ambrožič M, Gorjan L, Gomilšek M. Bend strength variation of ceramics in serial fabrication. J Eur Ceram Soc 2014, 34: 1873-1879.

[7] Saleh ME, Beuth JL, Boer MP. Validated prediction of the strength size effect in polycrystalline silicon using the three-parameter Weibull function. J Am Ceram Soc 2014, 
97: 3982-3990.

[8] Magnani G, Galvagno S, Sico G, et al. Sintering and mechanical properties of $\beta$-SiC powder obtained from waste tires. $J$ Adv Ceram 2016, 5: 40-46.

[9] Trustrum K, Jayatilaka ADS. On estimating the Weibull modulus for a brittle material. J Mater Sci 1979, 14: 1080-1084.

[10] Ritter JE, Bandyopadhyay N, Jakus K. Statistical reproducibility of the dynamic and static fatigue experiments. Am Ceram Soc Bull 1981, 60: 798-806.

[11] Bergman B. On the estimation of the Weibull modulus. J Mater Sci Lett 1984, 3: 689-692.

[12] Khalili A, Kromp K. Statistical properties of Weibull estimators. J Mater Sci 1991, 26: 6741-6752.

[13] Langlois R. Estimation of Weibull parameters. J Mater Sci Lett 1991, 10: 1049-1051.

[14] $\mathrm{Wu} \mathrm{D,} \mathrm{Zhou} \mathrm{J,} \mathrm{Li} \mathrm{Y.} \mathrm{Methods} \mathrm{for} \mathrm{estimating} \mathrm{Weibull}$ parameters for brittle materials. J Mater Sci 2006, 41: $5630-5638$.

[15] Tiryakioglu M. On estimating Weibull modulus by moments and maximum likelihood methods. J Mater Sci 2008, 43: 793-798.

[16] Bermejo R, Supancic P, Danzer R. Influence of measurement uncertainties on the determination of the Weibull distribution. J Eur Ceram Soc 2012, 32: 251-255.

[17] Bütikofer L, Stawarczyk B, Roos M. Two regression methods for estimation of a two-parameter Weibull distribution for reliability of dental materials. Dent Mater 2015, 31: e33-e50.

[18] Davies IJ. Unbiased estimation of Weibull modulus using linear least squares analysis-A systematic approach. $J$ Eur Ceram Soc 2017, 37: 369-380.
[19] Olkin I, Gleser LJ, Derman C. Probability, Models and Applications. New York: Macmillan Publishing Co., Inc., 1980.

[20] Gong J. A simple method for determining the Weibull estimator. J Mater Sci Lett 1997, 16: 875-876.

[21] Gong J, Li Y. Relationship between the estimated Weibull modulus and the coefficient of variation of the measured fracture strength for ceramics. J Am Ceram Soc 1999, 82: 449-452.

[22] Gong J. A new probability index for estimating Weibull modulus for ceramics with the least-square method. J Mater Sci Lett 2000, 19: 827-829.

[23] Song L, Wu D, Li Y. Optimal probability estimators for determining Weibull parameters. J Mater Sci Lett 2003, 22: 1651-1653.

[24] Nadarajah S, Kotz S. Comment on the probability indices. J Mater Sci 2006, 41: 6479-6480.

[25] Katayama Y, Hattori Y. Effects of specimen size on strength of sintered silicon nitride. J Am Ceram Soc 1982, 65: c164-c165.

[26] Xu Y, Cheng L, Zhang L, et al. Optimization of sample number for for Weibull functions of brittle materials strength. Ceram Int 2001, 27: 239-241.

Open Access The articles published in this journal are distributed under the terms of the Creative Commons Attribution 4.0 International License (http://creativecommons. org/licenses/by/4.0/), which permits unrestricted use, distribution, and reproduction in any medium, provided you give appropriate credit to the original author(s) and the source, provide a link to the Creative Commons license, and indicate if changes were made. 